\title{
Social Role
}

National Cancer Institute

\section{Source}

National Cancer Institute. Social Role. NCI Thesaurus. Code C25199.

The action or activity assigned to or required or expected of a person or group. 